\title{
Professional Psychology: Research and Practice
}

\section{Sustained Implementation of Cognitive-Behavioral Therapy for Youth Anxiety and Depression: Long-Term Effects of Structured Training and Consultation on Therapist Practice in the Field}

Brian C. Chu, Sofia Talbott Crocco, Cassidy C. Arnold, Ruth Brown, Michael A.

Southam-Gerow, and John R. Weisz

Online First Publication, October 13, 2014. http://dx.doi.org/10.1037/a0038000

CITATION

Chu, B. C., Talbott Crocco, S., Arnold, C. C., Brown, R., Southam-Gerow, M. A., \& Weisz, J. R. (2014, October 13). Sustained Implementation of Cognitive-Behavioral Therapy for Youth Anxiety and Depression: Long-Term Effects of Structured Training and Consultation on Therapist Practice in the Field. Professional Psychology: Research and Practice. Advance online publication. http://dx.doi.org/10.1037/a0038000 


\title{
Sustained Implementation of Cognitive-Behavioral Therapy for Youth Anxiety and Depression: Long-Term Effects of Structured Training and Consultation on Therapist Practice in the Field
}

\author{
Brian C. Chu and Sofia Talbott Crocco \\ Rutgers, The State University of New Jersey
}

\author{
Cassidy C. Arnold, Ruth Brown, and \\ Michael A. Southam-Gerow \\ Virginia Commonwealth University
}

\author{
John R. Weisz \\ Harvard University
}

\begin{abstract}
Identifying factors that promote sustained implementation of evidence-based treatments (EBTs) after therapists receive training is critical for professional psychology. To address the field's minimal knowledge in this area, we interviewed community-based therapists $(N=23)$ who had completed intensive training in cognitive-behavioral therapy (CBT) for either anxiety or depression as part of a randomized effectiveness trial (Southam-Gerow et al., 2010; Weisz et al., 2009). Therapists were interviewed 3 to 5 years after completion of the initial trial, representing one of the longest-term follow-ups of therapist practices after training. Therapists viewed each protocol and their individual CBT strategies as effective and appropriate for the majority of their current anxiety and depression caseloads. However, therapists used parts of each protocol much more frequently than the protocol as a whole (i.e., 78.5\% used parts of the Coping Cat, and 7.5\% used the whole protocol; $58.6 \%$ used parts of the PASCET, and $20 \%$ used the whole protocol). Therapists reported using problem-solving the most and exposure exercises the least for current anxious cases; they used cognitive restructuring the most and homework the least for current depression cases. Interventions that were more difficult to implement in usual care settings appeared less likely to be sustained. Future efforts should evaluate the characteristics and structure of EBTs that are most acceptable to therapists and should investigate which kinds of ongoing learning supports will maintain therapist skills in and continued use of EBTs.
\end{abstract}

Keywords: therapist training, dissemination, implementation

BRIAN C. ChU received his $\mathrm{PhD}$ in clinical psychology from Temple University. $\mathrm{He}$ is an associate professor and assistant department chair of clinical psychology at the Graduate School of Applied and Professional Psychology of Rutgers University. His areas of interest include transdiagnostic mechanisms and treatments for youth anxiety and depression, disseminating evidence-based care to community settings, and training issues.

Sofia TALBOTT CROcco received her PsyM in clinical psychology from the Graduate School of Applied and Professional Psychology of Rutgers University. She is currently working in private industry. Her interests include clinician training, dissemination and implementation, and community outreach to diverse populations.

CASSIDY C. ARNOLD received his MS in clinical psychology at Virginia Commonwealth University where he is continuing his studies as a clinical psychology doctoral candidate. He is currently completing his predoctoral clinical internship at the Medical University of South Carolina. His research interests include treatment integrity and dissemination and implementation science.

RUTH BROWN received her $\mathrm{PhD}$ in clinical child and adolescent psychology from Virginia Commonwealth University. She is currently a postdoctoral fellow at the Virginia Institute for Psychiatric and Behavioral Genetics at Virginia Commonwealth University. Her areas of professional interest include the assessment and treatment of anxiety and depression in people with Down syndrome and other intellectual and developmental disabilities.

Michael A. Southam-Gerow received his $\mathrm{PhD}$ in clinical psychology from Temple University. He is a professor and director of graduate studies in the psychology department at Virginia Commonwealth University. His research focuses on dissemination and implementation of evidence-based mental health assessment and interventions for children and families.

JoHN R. WeIsZ received his PhD in clinical and developmental psychology from Yale University. He is a professor in the Department of Psychology at Harvard University and a professor at Harvard Medical School. His work focuses on developing and testing strategies for implementing and sustaining evidence-based interventions within everyday clinical care and educational settings for children and adolescents. His broad goal is to find effective ways to put science into practice and to improve mental health of children and teens.

THIS RESEARCH WAS SUPPORTED in part by Grant R01-MH574347 from the National Institutes of Mental Health to John R. Weisz. We thank the therapists who provided their valuable feedback for this project.

CORRESPONDENCE CONCERNING THIS ARTICLE should be addressed to Brian C. Chu, Department of Clinical Psychology, GSAPP, Rutgers University, 152 Frelinghuysen Road, Piscataway, NJ 08854. E-mail: brianchu@rci.rutgers.edu 
What happens to evidence-based practice after intensive training and consultation supports dwindle? Despite a substantial knowledge base supporting the efficacy of psychological interventions in the treatment of child and adolescent (herein referred to as "youth") emotional and behavioral disorders (David-Ferdon \& Kaslow, 2008; Silverman et al., 2008), disseminating evidencebased treatments (EBTs) in a way that leads to sustained implementation in community settings remains a vexing challenge. Little is known about how well EBT training holds up over time or what factors influence their sustainability in local practice. Typical protocols for training therapists in evidence-based treatments usually include intensive didactic or experiential workshops and close supervision. However, relatively few efforts have been made to follow up with therapists to determine how well the skills have been sustained over time. And yet, therapist attitudes, particularly those who have had direct experience with implementing novel interventions, are highly influential in determining therapist interest and willingness in maintaining skills as intended (Aarons, 2004; Borntrager, Chorpita, Higa-McMillan, \& Weisz, 2009; Nakamura, Higa-McMillan, Okamura, \& Shimabukuro, 2011). Several studies have demonstrated the relevance of attitudes on therapist use of EBTs (e.g., Beidas \& Kendall, 2010; Henggeler et al., 2008; Jensen-Doss, Hawley, Lopez, \& Osterberg, 2009). For example, Henggeler et al. (2008) found that implementation of contingency management interventions after a 1-day workshop was predicted by therapist positive attitudes toward behavior therapy and beliefs about the efficacy of the intervention.

Therapist training programs have come to reflect a wide range of activities from bibliographic training (e.g., self-instruction using treatment manuals) to expert-led didactics (e.g., continuing education seminars) to ongoing consultation and supervision from experts. The most common method of training therapists new treatment approaches (i.e., reading written materials and attending single-event or limited workshops) tends to have little effect in producing desired outcomes, such as, increased skill or competence in EBT strategies (Beidas \& Kendall, 2010; Herschell, Kolko, Baumann, \& Davis, 2010). Adding ongoing supervision, feedback, or extended coaching tends to enhance retention of skill after initial training workshops (Beidas \& Kendall, 2010; Herschell, Kolko, Baumann, \& Davis, 2010). In one randomized trial of training approaches, community therapists' ability to implement CBT was higher at 3-month follow-up when training workshops were augmented by ongoing supervisory supports than when no such supports were provided (Sholomskas et al., 2005). In another investigation of multiple training methods of Motivational Interviewing skills, Miller, Yahne, Moyers, Martinez, and Pirritano (2004) found no skill improvements through self-study and only modest gains in skills after clinicians received a 2-day workshop; and therapist skills returned to baseline levels after 4 months without further supports. However, the addition of periodic feedback and/or coaching improved retention of proficiency up to 12 months after initial training. Similarly, Beidas, Edmunds, Marcus, and Kendall (2012) found that the number of consultation hours after training significantly predicted higher therapist adherence and skill 3-months after training. Such results suggest that ongoing supervisory supports are necessary after initial training to promote successful short-term skill attainment, a necessary precursor to long-term treatment implementation.
Little is known about the long-term effects of these implementation supports, because few implementation studies include follow-up assessments. In two reviews of EBT training methods (Beidas \& Kendall, 2010; Herschell et al., 2010), the majority of studies only evaluated immediate posttraining effects of the training program, including outcomes such as, therapist knowledge, skill, and attitudes. In Beidas and Kendall's review, only $40.6 \%$ of training evaluations conducted longer-term follow-ups, wherein most follow-ups $(61.5 \%)$ were 6 months or shorter, and all were shorter than a year after training. In Herschell et al.'s review, using different study inclusion criteria, $32.7 \%$ of studies conducted longer-term follow-ups, of which, $61.1 \%$ were 6 months or shorter. In one of the few longer-term follow-up studies, Saitz, Sullivan, and Samet (2000) completed a naturalistic follow-up of trainees who had attended a half-day continuing education course on substance abuse screening and brief intervention. Trainees were phone interviewed up to 5 years after attending the workshop. Most (96\%) reported the workshop made a difference in their clinical practice, and the large majority of professionals (86\% to $96 \%$ ) reported they were more likely to use formal screening and counseling procedures taught in the workshop. The study lacked a control group and participants self-selected themselves to attend the workshop. Nevertheless, longer-term follow-up studies like this provide an indication that relatively brief trainings can impact professional report of services.

The Beidas and Kendall (2010) and Herschell et al. (2010) reviews also identified few training studies of youth protocols with long-term follow-up. No studies conducted follow-up assessments beyond 1 year, and most were between 2 and 6 months. For example, Henggeler et al. (2008) conducted 6 months of naturalistic follow-ups after public sector practitioners attended a 1-day workshop. At 6 months, 58\% of workshop attendees had attempted to use the targeted intervention (contingency management) in substance-abusing adolescents they were treating. In a controlled trial of a school-based suicide-risk identification training program, Wyman et al. (2008) demonstrated that a 1.5-hr training plus 30 -min refresher course could produce significant changes in increased knowledge and provider appraisals a year after training. However, the training program did not increase targeted behaviors (e.g., asking students about suicide; referral behaviors) compared with the control condition, and targeted behaviors only increased for the $14 \%$ of school staff who had already been communicating with students about suicide before the training.

In another comparison trial, Beidas et al. (2012) compared three types of brief training approaches (6-hr standard workshop, computer-based workshop, and an augmented, experiential workshop) plus 3 months of weekly phone consultation in training providers in CBT for anxious youth. The brief training programs had the most immediate impact on knowledge, but the majority of participants also achieved "training criterion" in adherence $(61 \%)$ and CBT skills $(85 \%)$ by the end of the 3 -month phone consultation period. Together, the child training literature provides evidence for both encouragement and concern. Wyman et al.'s (2008) findings suggest that brief exposure to training materials is insufficient to sustain targeted behaviors over the longer-term, and Beidas et al.'s findings suggest that continued consultation is essential for improving poor rates of sustained skills. At the same time, Henggeler et al. (2008) suggests that some therapist behaviors can be impacted by relatively small doses of training. Each 
study is limited by its relatively brief follow-up period. We have less information about how effects would be sustained beyond 6 to 12 months after the intensive training period ends.

The current study examines community therapists' use of two CBT protocols and their attitudes toward using EBTs in two clinical contexts: treating anxious and depressed youth as part of a community research study and treating youth in their current practice setting. Structured interviews were completed with clinicians 3 to 5 years after they had served as study therapists in a community-based randomized effectiveness trial for which they had received intensive workshop training and supervisory supports (Southam-Gerow et al., 2010; Weisz et al., 2009). Such training and consultation represented a higher intensity of training than has been offered in past investigations of therapist training. The goal was (a) to determine whether community therapists continued to implement the EBTs on their own after the intensive supports of the research study ended, and (b) to assess therapist attitudes toward the EBT (appropriateness, difficulty implementing, and effectiveness) after having the opportunity to implement the protocols in both a clinical trial and in their current practice. It was expected that attitudes toward EBT and specific treatment protocols would remain positive, given past research that suggests positive attitudes can develop even after brief training. It was also expected that therapists would report using CBT techniques in their practice, particularly the specific interventions in which they received training. However, it was expected that some strategies would be easier to sustain than others. Use of didactic components, such as emotion education, problem solving, and cognitive restructuring, was expected to be sustained relatively equally over the follow-up period. However, we expected in vivo exposures to be more difficult to sustain, as this intervention may require sustained practice and external supports. In vivo exposures tend to require greater availability of resources (e.g., exposure confederates, a team of like-minded therapists) and flexibility in practice setting (e.g., ability to conduct sessions outside) that might challenge their implementation.

\section{Method}

\section{Participants and Procedures}

The Youth Anxiety and Depression Study (YADS; SouthamGerow et al., 2010; Weisz et al., 2009) was the first fully randomized effectiveness study examining CBT for youth anxiety and depression in clinically representative treatment settings. As part of the YADS study, community clinicians from outpatient public mental health clinics were trained to deliver one of two manualbased CBT treatments for anxiety or depression in children (Coping Cat; Kendall \& Hedtke, 2006; Primary and Secondary Control Enhancement Therapy [PASCET]; Weisz, Thurber, Sweeney, Proffitt, \& LeGagnoux, 1997). A double randomization procedure was used whereby therapists were randomly assigned to be trained in CBT or to conduct treatment-as-usual, and participating youth were randomly assigned to CBT or treatment-as-usual. Training and supervisory supports were consistent with the highest standards of the training literature (Beidas et al., 2010, 2012; Herschell et al., 2010). Therapists attended a 6-hr training workshop that included didactics and group role plays. When treating a YADS case, therapists received weekly supervision that included feedback on video or audiotaped sessions.

All therapists who had received training in CBT (Coping Cat or PASCET) as a study therapist on YADS were eligible and invited to participate via phone and/or email. The YADS trial was completed in 2004 and follow-up interviews were conducted from 2007-2009 (considerable time was required to locate therapists and to find acceptable times for interviews to be conducted). For therapists no longer at their original work sites, contact information was obtained from searches of state licensing boards and/or referral from coworkers. Therapists were provided with study information and then provided oral consent to participate. All procedures were approved by the Virginia Commonwealth University Institutional Review Board. Eleven of 18 (61.1\%) therapists trained in the Coping Cat, and 12 of 26 (46.2\%) therapists trained in PASCET agreed to complete current study procedures. The remaining therapists were not able to be contacted (e.g., out-of-date contact information; $n=12$ ) or did not respond to recruitment efforts $(n=6)$. Web-based versions of the survey were also developed to accommodate therapist schedules. Table 1 summarizes participant characteristics.

\section{Measures}

Therapist follow-up Coping Cat Interview. The Coping Cat Interview (CCI; available from authors) is a structured interview designed to assess a clinician's perception of the Coping Cat's utility across multiple settings. It can be administered by phone, mail, or Web-based survey. The CCI contains two sections. First, therapists are asked to rate the effectiveness of and difficulty in implementing the Coping Cat in the anxiety cases they saw as part of the YADS trial. Therapists are asked to consider each aspect for the Coping Cat protocol as a whole and then to consider each aspect for the Coping Cat's individual treatment components: Emotion Education, Relaxation, Cognitive Restructuring, Problem Solving, Self-Evaluation and Rewards, Role Play, In Vivo Exposure, and Homework. Items are rated on a 10-point Likert-type scale from $0=$ Not at all [effective/difficult] to $4=$ Moderately, to $9=$ Extremely [effective/difficult]. For example, therapists are asked, "Thinking about your time in the YADS study, how effective were the following Coping Cat components for your project cases? How effective was [Relaxation]?"

Second, therapists are asked to evaluate the Coping Cat for its use in cases seen in the therapist's current clinical setting. Therapists are asked to rate the Coping Cat's effectiveness, difficulty in implementing, and appropriateness for current or recent cases where anxiety was a clinical focus. Each item is again rated on a 0 to 9 scale and respondents rate both the overall treatment protocol and individual treatment components. They then estimate the percentage of their current anxiety cases in which they have actually used the Coping Cat. Finally, therapists estimate the percentage of cases they used the Coping Cat (in part or in whole) across a broad range of nontargeted clinical problems (attention deficit hyperactivity disorder [ADHD], defiance, family relationship problems, and other) to examine perceived generalizability of the protocol.

Therapist follow-up PASCET Interview. The PASCET Interview (PI; available from authors) parallels the CCI structurally and assesses the same domains of therapist perceptions across 
Table 1

Participants Demographics and Professional Setting

\begin{tabular}{lll}
\hline & Coping Cat Therapists $(N=11)$ & PASCET Therapists $(N=12)$ \\
\hline Age: $M(S D)$ & $45.22(13.85)$ years & $37.64(5.64)$ years \\
& Range: $31-76$ & Range: $32-51$ \\
Gender & $9(82 \%)$ female & $8(67 \%)$ female \\
Ethnicity & $5(45 \%)$ White & $7(58 \%)$ White \\
& $3(27 \%)$ not reported & $5(42 \%)$ Latino/Latina \\
& $1(9 \%)$ Latino/Latina & \\
& $1(9 \%)$ Asian & \\
& $1(9 \%)$ multiethnic & $6(50 \%)$ Ph.D. \\
Highest degree obtained & $6(55 \%)$ Psy.D. & $3(25 \%)$ Psy.D. \\
& $3(27 \%)$ MSW/LCSW & $2(16 \%)$ MSW/LCSW \\
& $2(18 \%)$ Master's in Psychology & $1(8 \%)$ Master's in Psychology \\
Years of clinical practice & $13.09(11.53)$ years; Range: $4-45$ & $5.50(5.05)$ years \\
& & Range: 2-20 \\
Current employment setting & $4(36 \%)$ private practice & $4(33 \%)$ private practice \\
& $3(27 \%)$ community mental health & $3(25 \%)$ community mental health \\
& $2(18 \%)$ school & $2(17 \%)$ University setting \\
& $1(9 \%)$ hospital & $2(17 \%)$ other \\
Method of data collection & $1(9 \%)$ other & $1(8 \%)$ school \\
& $6(55 \%)$ via phone interview & $6(50 \%)$ via phone interview \\
& $5(45 \%)$ via online survey & $6(50 \%)$ via online survey \\
\hline
\end{tabular}

YADS and current depressed cases: effectiveness and difficulty in implementing the PASCET as a whole and for its individual components, appropriateness for depressed youth and others, and percentage of current cases for whom they have actually used the PASCET. Individual PACET components included, Problem Solving, Activity Selection, Relaxation, Presenting a Positive Self, Talent Development, Cognitive Restructuring, Social Support, Sequential Coping, and Homework. Items are rated on a 10-point Likert-type scale from $0=$ Not at all [effective/difficult/appropriate] to $4=$ Moderately, to $9=$ Extremely [effective/difficult/ appropriate].

\section{Results}

\section{Initial CC Adherence}

Initial therapist adherence was strong for therapists trained in the CC protocol (Southam-Gerow et al., 2010) with integrity ratings indicating that $98.9 \%(S D=3.77$, range: $87.5-100.0 \%)$ of $\mathrm{CC}$ sessions contained the expected procedures.

\section{Perceived Effectiveness and Difficulty in Implementing CC Strategies}

Table 2 summarizes therapist attitudes toward the CC and its strategies. For cases seen in the YADS trial, all CBT techniques were seen as effective for anxiety cases. On a 0-9 scale, perceived effectiveness scores ranged from 5.3 to $7.1(M=6.5, S D=.56)$, placing the mean at a level greater than "moderately" effective (based on CCI anchors given to respondents). Homework was seen as the least effective and role plays were seen as the most effective. Mean effectiveness for the whole CC protocol was $6.7(S D=1.9)$ for YADS cases. Perceived difficulty in implementing specific CBT techniques with YADS cases was low, with scores ranging from 3.2 to $4.5(M=3.7, S D=.46)$, where a score of 4 equated to moderately difficult. The least difficult technique to implement was rewards, and the most difficult was exposure. Overall, the CC protocol as a whole was not seen as difficult to implement in the context of the YADS trial $(M=3.8, S D=1.4)$.

Greater variation was evident in therapist attitudes regarding current casework with anxious youth. Most CBT techniques were still seen as effective $(M=6.1, S D=1.3$, range: $4.3-7.2)$. However, perceived effectiveness of exposures was $37 \%$ lower for current cases than for YADS cases (paired $t$ test $=3.24, p=.02$ ), and $7 \%$ lower for role plays (paired $t$ test $=2.75, p=.02$ ). Perceived effectiveness for other strategies were statistically nonsignificantly different (all paired $t$ tests, $p>.05$ ). The overall perceived effectiveness of the CC protocol was $34 \%$ lower for current cases compared with YADS cases but this difference was nonsignificant, paired $t=1.45, p=.22$. Perceived difficulty implementing $\mathrm{CC}$ techniques with current anxious cases was relatively similar to YADS cases $(M=3.5, S D=1.0$, range: 2.7-5.0), and exposures were again perceived as the most difficult technique to implement. Nonsignificant differences were found in paired $t$ tests across strategies comparing YADS and current cases (all paired $t$ tests, $p>.05$ ). Overall, perceived difficulty in implementing the entire protocol was $26 \%$ greater for current cases but this difference was statistically nonsignficant (paired $t$ test $=-1.62, p=.14$ ).

\section{Perceived Utility and Use of CC Strategies With Current Anxiety Cases}

Therapists were asked for what percentage of their current anxiety caseload each CC strategy was appropriate, and for what percentage of their current anxiety caseload the $\mathrm{CC}$ was actually used (see Table 2). A wide range of responses was evident with exposures seen as appropriate for the smallest percentage of current anxiety cases $(M=45.9 \%, S D=21.5 \%)$ and problem solving seen as appropriate for the highest percentage $(M=87.3 \%, S D=$ 


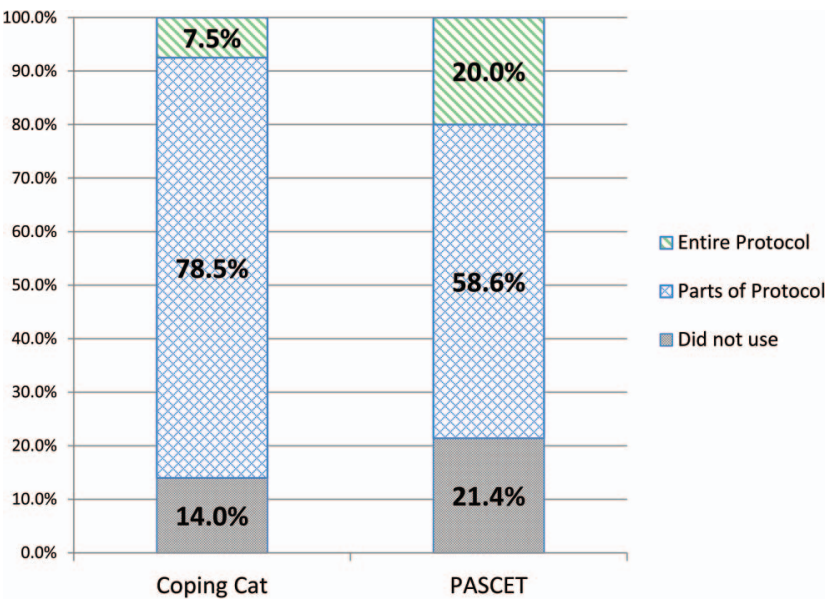

Figure 1. Practitioner use of CBT for youth anxiety and depression 3 to 5 years after completing training and consultation as part of a randomized effectiveness trial. Data represent the proportion of current cases with whom therapists reported "actually using" none of, parts of, or the entirety of the Coping Cat or PASCET protocols. See the online article for the color version of this figure.

15.6\%). The entire CC protocol was seen as appropriate for $44.5 \%$ $(S D=19.5 \%)$ of anxiety cases, and parts of the CC protocol were seen as appropriate for $89.5 \%(S D=12.6 \%)$.

Therapist use of CC strategies with current anxiety cases ranged from exposures $(M=34.5 \%, S D=22.9 \%)$ to problem solving $(M=85.0 \%, S D=15.1 \%)$. Therapists reported using the entire CC protocol with $7.5 \%$ of anxiety cases $(S D=16.2 \%)$ and parts of the CC protocol with $78.5 \%(S D=27.1 \%)$. Figure 1 illustrates the distribution of practitioner use of the $\mathrm{CC}$.

\section{Generalizability of CC Protocol}

Therapists were asked to what extent the $\mathrm{CC}$ or its parts were effective for, and used with, clinical problems that are not specifically targeted by the $\mathrm{CC}$ (see Table 3 ). Therapists reported the $\mathrm{CC}$ was broadly effective for much of their nonanxious caseloads $(M=5.7, S D=.52$, range: $5.2-6.5)$. Therapists reported using at least parts of $\mathrm{CC}$ in $64.5 \%$ of cases with depression, $44.5 \%$ of cases with ADHD, $37.5 \%$ of cases with defiance or behavioral problems, $47.5 \%$ of cases with family relationship problems, and $27.1 \%$ of cases with "other" problems.

\section{Initial Adherence of PASCET}

Initial therapist adherence was strong for therapists trained in the PASCET protocol (Weisz et al., 2009) with integrity ratings indicating that $98.0 \%(S D=0.01$, range: $88.0-100.0 \%)$ of PASCET sessions contained a required element.

\section{Perceived Effectiveness and Difficulty in Implementing PASCET Strategies}

Table 4 summarizes therapist attitudes toward the PASCET and its strategies. For depression cases seen in the YADS trial, all CBT techniques were seen as effective. On a 0-9 scale, perceived effectiveness scores ranged from 5.3 to $7.0(M=6.3, S D=.60)$, 
Table 3

Applicability of the Coping Cat (CC) and PASCET to Nontargeted Clinical Problems

\begin{tabular}{|c|c|c|}
\hline & $\begin{array}{c}\text { Whole or } \\
\text { Parts of CC } \\
M(S D)\end{array}$ & $\begin{array}{c}\text { Whole or Parts of } \\
\text { PASCET } \\
M(S D)\end{array}$ \\
\hline \multicolumn{3}{|l|}{ Depression } \\
\hline Effective for this problem? (0-9) & $6.5(1.51)$ & \\
\hline Percentage of cases protocol was used for depression & $64.5 \%(24.99)$ & \\
\hline Modal response (\% of cases used) & $50 \%$ & \\
\hline \multicolumn{3}{|l|}{ Anxiety } \\
\hline Effective for this problem? (0-9) & & $5.8(2.91)$ \\
\hline Percentage of cases protocol was used for anxiety & & $37.3 \%(41.74)$ \\
\hline Modal response ( $\%$ of cases used) & & $0 \%$ \\
\hline \multicolumn{3}{|l|}{ ADHD } \\
\hline Effective for this problem? (0-9) & $5.2(2.25)$ & $5.5(3.79)$ \\
\hline Percentage of cases protocol was used for ADHD & $44.5 \%(30.95)$ & $23.0 \%(35.29)$ \\
\hline Modal response ( $\%$ of cases used) & $20 \%$ & $0 \%$ \\
\hline \multicolumn{3}{|l|}{ Defiance/behavioral problems } \\
\hline Effective for this problem? (0-9) & $5.3(1.73)$ & $5.4(2.94)$ \\
\hline Percentage of cases protocol was used for Defiance & $37.5 \%(27.61)$ & $27.0 \%(32.68)$ \\
\hline Modal response ( $\%$ of cases used) & $20 \%$ & $0 \%$ \\
\hline \multicolumn{3}{|l|}{ Family relationship problems } \\
\hline Effective for this problem? (0-9) & $5.9(1.29)$ & $4.3(2.71)$ \\
\hline $\begin{array}{l}\text { Percentage of cases protocol was used for family } \\
\text { relationship problems }\end{array}$ & $47.5 \%(26.6)$ & $27.7 \%(31.5)$ \\
\hline Modal response (\% of cases used) & $40 \%$ & $0 \%$ \\
\hline \multicolumn{3}{|l|}{ Other } \\
\hline Effective for this problem? (0-9) & $5.6(1.52)$ & $6.2(3.63)$ \\
\hline $\begin{array}{l}\text { Percentage of cases protocol was used for other } \\
\text { problems }\end{array}$ & $27.1 \%(36.84)$ & $22.73 \%(39.07)$ \\
\hline Modal response (\% of cases used) & $0 \%$ & $0 \%$ \\
\hline
\end{tabular}

where a score of six equated to a rating higher than moderately effective, based on PI anchors given to respondents. Relaxation and sequential coping (preplanning multiple solutions to a problem and using continuous assessment to determine which one works) were seen as the least effective, and problem solving was seen as the most effective. Mean effectiveness for the whole PASCET protocol was $6.4(S D=2.4)$ for YADS cases. Perceived difficulty in implementing specific CBT techniques with YADS cases was low, with scores ranging from 2.9 to $4.5(M=3.5, S D=.67)$, where a score of four equated to moderately difficult. The least difficult techniques to implement were problem solving and cognitive restructuring (identifying and challenging negative thoughts), and the most difficult was assigning homework. The PASCET protocol as a whole was not seen as difficult to implement in the context of the YADS trial $(M=2.2, S D=1.9)$.

Similar attitudes were reported for current depression cases. Most techniques were viewed as effective for current depression cases $(M=6.5, S D=.73$, range: $5.3-7.1)$, and perceived effectiveness ratings of relaxation were $32 \%$ greater for current compared with YADS cases (paired $t=-2.35, p=.05$ ). All other paired $t$ tests were statistically nonsignificantly different, $p>.05$. Perceived effectiveness of the whole PASCET was $20.3 \%$ greater for current depression cases, but this difference was nonsignificant, $t=-2.00, p=.18$. Perceived difficulty implementing PASCET appeared to be lower across techniques $(M=2.6, S D=$ 95, range: $1.8-3.6)$ when comparing current depression cases to YADS cases. Homework continued to be the most difficult strategy to implement and assigning activities the easiest. Implementation was rated as relatively easier in current depression cases, compared to YADS cases, in relaxation, $t=2.16, p=.05$, self-presentation, $t=2.49, p=.03$, social support, $t=4.00, p=$ .003 , and sequential coping, $t=4.67, p=.001$. However, perceived difficulty in implementing the entire PASCET protocol was $122.7 \%$ greater in current depression casework (paired $t=-3.22$, $p=.01)$.

\section{Perceived Utility and Use of PASCET Strategies With Current Depression Cases}

Therapists were asked for what percentage of their current depression caseload that each PASCET strategy was appropriate, and in what percentage of their current depression caseload they used PASCET strategies (see Table 4). Homework was seen as appropriate for the smallest percentage of current depression cases $(M=58.2 \%, S D=25.4 \%)$ and cognitive restructuring was seen as appropriate for the highest percentage $(M=90.4 \%, S D=$ $12.1 \%)$. The entire PASCET protocol was seen as appropriate for $44.6 \%(S D=35.3 \%)$ of depression cases, and parts of the PASCET protocol were seen as appropriate for $85.4 \%$ ( $S D=$ $19.9 \%)$.

Therapist use of PASCET strategies with current depression cases ranged from assigning homework $(M=23.9 \%, S D=$ $33.9 \%)$ to cognitive restructuring $(M=70.5 \%, S D=38.2 \%)$. Therapists reported using the entire PASCET protocol with $20 \%$ of depression cases $(S D=39.1 \%)$ and parts of the PASCET protocol with $58.6 \%(S D=40.6 \%)$. Figure 1 illustrates the distribution of practitioner use of the PASCET. 


\section{Generalizability of the PASCET Protocol}

Therapists were asked to what extent PASCET or its parts were effective for, and used with, clinical problems that are not specifically targeted by PASCET (see Table 3 ). Therapists reported that PASCET was moderately effective for much of their nondepression caseloads $(M=5.4, S D=.71$, range: $4.3-6.2)$. Therapists reported using at least parts of PASCET in $37.3 \%$ of cases with anxiety, $23.0 \%$ of cases with ADHD, $27.0 \%$ of cases with defiance or behavioral problems, and $27.7 \%$ of cases with family relationship problems.

\section{Discussion}

As the field turns greater attention from evidence-based treatment development to evidence-based provider training, it is becoming increasingly important to incorporate feedback from all potential consumers. In this case, we endeavored to learn from a unique set of professionals - mental health providers who had the opportunity to implement a novel evidence-based protocol in two settings, a structured randomized trial and in a therapist's own naturalistic practice. Three to 5 years after completing intensive training in two CBT protocols that included weekly consultation, therapists continued to report relatively positive attitudes and continued use of both protocols across a range of clinical settings. However, in current casework, therapists tended to use parts of each protocol rather than implementing the entire program, and some specific strategies (e.g., exposure and homework) were less used than others.

Therapists reported that they used the majority of CBT techniques from the $\mathrm{CC}$ and PASCET protocols with their current anxiety and depression cases. Therapists considered most techniques generally effective and appropriate for the majority of their anxious and depressed clients. Therapists perceived the difficulty of implementing these practices in their current contexts as low. Therapists reported "actually using" at least parts of the CC in $78.5 \%$ of current anxiety cases and parts of the PASCET in $58.6 \%$ of current depression cases. More specifically, therapists reported using six of eight CC techniques in over $60 \%$ of their current anxiety cases. Therapists reported using six out of nine PASCET techniques in over $50 \%$ of their depression cases. Given the significant investment required to provide even initial training in novel therapy skills, it is heartening to see therapists appreciating and using EBT techniques after initial training. By comparison, Jensen-Doss et al. (2009) conducted a review of child mental health practice in Texas after provision of state-mandated evidence-based treatment training. There, $72 \%$ of child mental health providers reported using a mandated evidence-based proto$\mathrm{col}$ in $75 \%$ of their cases or more. Sustainability rates in the current study were somewhat lower, but the fact that YADS therapists reported using CBT techniques in 50-60\% of their anxiety and depression cases on a volunteer basis is encouraging news.

Perceived generalizability of the two protocols provided another sign of promise. The CC is a "single target" treatment protocol, but therapists endorsed that at least parts of the $\mathrm{CC}$ were useful for a wide range of youth problems, including depression (appropriate for $64.5 \%$ of cases), $\mathrm{ADHD}(44.5 \%)$, defiance $(37.5 \%)$, and family relationship problems $(47.5 \%)$. The PASCET was also seen as broadly applicable to a diversity of problems, including anxiety (37.3\% of cases), ADHD (23\%), defiance (27\%), and family 
relationship problems $(27.7 \%)$. The perception of broad applicability of these protocols to multiple target problems bodes well for their long-term sustainability in community settings where case complexity may be seen as a barrier to EBT implementation. The ability to treat many problems with a single set of techniques and treatment aids (manual, workbook) simplifies the task of choosing and implementing interventions.

Important lessons were also learned from therapist feedback. Not all CBT techniques were viewed as equally positive. By a large margin, therapists preferred to use parts of the two protocols over the entire protocol. Therapists used parts of the CC in $78.5 \%$ of current anxiety cases compared with the $7.5 \%$ of cases in which they used the entire protocol. Parts of the PASCET were used in $58.6 \%$ of depression cases compared with $20.0 \%$ of cases for the entire PASCET. This is consistent with past research where clinicians expressed preference for modular-based formats and selfselecting specific interventions based on client need (Borntrager et al., 2009; Palinkas et al., 2008). Past research has shown that therapists do not harbor negative attitudes toward evidence-based practice so much as negative impressions of treatment manuals and their implied rigidity. Our findings confirm that therapists made independent decisions about treatment selection. Whether these decisions are based on preexisting therapist attitudes, prior experiences with evidence-based treatments, or in the nature of the original training remains to be seen. All of these factors may play a role (Jensen-Doss et al., 2009; Nakamura et al., 2011).

Whether it is a positive or negative outcome that therapists used parts, rather than the entirety, of EBT manuals is the subject of ongoing debate. On one hand, one study found that modular-based treatments may perform better than standard protocols focused on single disorders or homogeneous clusters (Weisz et al., 2012). In this study, the modular condition even promoted the use of more evidence-based content than usual care treatment and more nonspecific therapy content than standard sessions. This suggests that modular formats might strike the balance of structure and flexibility. On the other hand, research exists to suggest that permitting "too much" flexibility in clinical decision making can lead to "straying" from recommended EBT interventions (Craske et al., 2007). The current study remains neutral in this debate as the design permits purely descriptive interpretations. Our goal was to learn from therapists which practices were naturally seen as most effective and easy to implement and which might require greater supports should increasing their use be a goal.

Differing attitudes emerged regarding specific CBT strategies when comparing use in a clinical trial or current practice. Exposures and role plays in the CC were seen as less effective for therapists' current anxiety caseloads, and exposures continued to be rated as the most difficult intervention to implement. Indeed, therapists reported using exposures in only $34.5 \%$ of current anxiety cases while using all other CC strategies in over $60 \%$ of cases. Homework, perhaps surprisingly, was only used in $43.5 \%$ of current anxiety cases. None of the individual PASCET techniques were seen as less effective or more difficult to implement in current clinical practice. In fact, several strategies were seen as easier to implement in their current depression cases than during the YADS trial, including, relaxation, self-presentation skills, seeking social support, and training sequential coping (i.e., the use of contingency coping strategies). Relaxation was also perceived as more effective with current depression cases. However, per- ceived difficulty in implementing the entire PASCET protocol was greater in current depression cases compared with YADS cases. Further, most individual PASCET techniques were actually used in under $60 \%$ of current depression cases, with homework used in under $25 \%$ of depression cases. The one notable exception was cognitive restructuring which therapists reported using in $70 \%$ of their current depression cases.

Several themes emerge from these reports. The techniques therapists reported using most appear to be those that fit well within traditional clinical care settings. Cognitive restructuring was among the most frequently used interventions (73\% of CC; $70 \%$ of PASCET). Therapists reported using emotion education, relaxation, problem-solving, and rewards assignment in at least $75 \%$ of anxiety cases. Each of these interventions fits easily into traditional one-on-one, office-based talk therapy and blends a combination of didactics, discussion, and mild behavioral rehearsal.

Action-oriented, behavioral interventions, such as exposure and homework were less frequently implemented, a worrisome finding, given the evidence-base that supports in vivo exposure and extratherapy practice (homework) as potent components of treatment (David-Ferdon \& Kaslow, 2008; Silverman et al., 2008). Exposure and other experiential interventions pose several challenges for therapists in traditional clinic settings. In vivo exposures can require substantial resources to maximize their benefit, including supplies, confederate actors, an ability to travel to naturalistic sites, and sufficient time for set-up, enactment, and postprocessing. Such demands pose challenges to common clinic structures where session time may be restricted to 50-min sessions and reimbursement schedules do not account for prolonged sessions or pay for supplies. Therapists working in relative isolation may find it difficult to find role playing confederates-either fellow professionals who will be prepared to handle youth conducting exposures throughout the clinic or same-aged peers to serve as mutual exposure participants. Clinic policies and liability concerns may limit a therapist to conducting therapy in the therapy room or clinic, limiting the ability to create naturalistic exposures. Each of these barriers, among others, need to be considered and addressed as we attempt to implement exposure exercises in local practice.

Homework was a second technique significantly underutilized in current practice. It was considered less appropriate, less effective, and more difficult to implement with anxious and depressed youth than other strategies. Previous research suggests that clinicians tend to implement techniques that they believe are effective and enjoyable (Cook, Schnurr, Biyanova, \& Coyne, 2009). Thus, therapist attitudes toward homework may reflect the challenges in getting youth to complete homework more than beliefs about the strategy, itself. This may be unfortunate given the apparent importance of homework in extending CBT lessons outside the therapy room (Kazantzis, Whittington, \& Dattilio, 2010).

These results suggest that treatment approaches that do not fit smoothly into real-world conditions of clinical practice may be less likely to be sustained over time than interventions that do not clash with everyday practice conditions or constraints. This is consistent with the recent analysis by Weisz, Ugueto, Cheron, and Herren (2013) suggesting that everyday practice exists within a kind of "mental health ecosystem," and that the match or mismatch between treatment characteristics and facets of the ecosystem will strongly influence whether the practices can be implemented and sustained. In cases where certain treatment components that are 
important to successful outcomes clash with the ecosystem, it may be critical to structure and deliver those components to fit more smoothly into the conditions of everyday clinical practice.

A second direction for future work involves enhancing ongoing support for implementation efforts. A number of models have been offered to extend training to local practice settings (SouthamGerow, Rodriguez, Chorpita, \& Daleiden, 2012). These include pyramid training (train-the-trainer, cascading), academic detailing (pairing novice and expert mentors), and pairing training efforts to therapist characteristics (prior training background, theoretical orientation, familiarity with EBTs). Of course, these are intensive (and expensive) training approaches. Costs may be reduced through the use of technology (Beidas, Koerner, Weingardt, \& Kendall, 2011), such as, supplying printable worksheets online or making video-based demonstrations available at the time of implementation can help reduce barriers at the point of clinical contact. Expert consultation can also be made more cost-effective through technology. Fostering chat-rooms and wiki sites that allow experts to consult with a larger group of providers can maintain therapist interest and confidence. A newer movement entails the development of "peer learning communities" that leverage the interests and resources among local groups of practitioners to facilitate self- and collaborative-learning (Darling-Hammond \& Richardson, 2009; Tosey, 1999). Experimentation with this model has primarily occurred in education and corporate settings and is only now being considered as a viable method for sustaining therapist learning in therapy practice (Chu, Carpenter, Wyszynski, Conklin, \& Comer, 2014).

The current study has limitations. The sample of participating therapists was small and represented only a portion of the original group of YADS therapists. Nonparticipating therapists may have had different opinions or may have been less inclined to maintain EBTs. Therapists were trained in either CC or PASCET but not both, so comparisons across the two treatments were limited. In the original YADS study, therapists were randomly assigned to receive training in either PASCET, CC, or to a usual care condition, so we would expect that each group of therapists reflects typical and equivalent groups of professionals. Further, we did not maintain control over therapists after the YADS study ended; therapists could have self-studied or discussed opinions about CC or PASCET with other study therapists. This lack of control may have led to some contamination, but it does represent the natural course of learning after any form of training. The results are based solely on therapist report, some of which were retrospective in nature. Therapist perspective carries significant weight in this line of research, but future research would be bolstered by including objective data (e.g., chart review; fidelity ratings of video recorded sessions). Interviews were conducted over a long period of time (3-5 years after study termination), but the small sample prevented analysis to examine relation between therapist report and latency between original study and follow-up interview. It should be noted that therapists had participated in more intensive training and consultation than is typically available; effects may not generalize to lower dose efforts. We also encourage the study of therapist training and consultation programs across theoretical orientations. Such comparative work might illuminate common and specific training elements that support evidence-based practice.

The methodological strengths of the study are that community practitioners were participants rather than research employees or graduate student trainees. Therapists were employees of community mental health clinics who were seeing cases as part of their natural caseloads. Therapists then had the unique experience of implementing CBT protocols in the context of a randomized effectiveness trial (with ongoing supervisory supports) and then later in their current clinical context (that represented a range of clinical settings). Our longer term follow-up (3-5 years) is the longest we could identify in the youth training literature. This provides a particularly useful test of the holding power of EBTs over time in everyday practice.

The results of this study encourage both optimism and caution for those engaged in dissemination and implementation efforts. Community therapists can be trained to implement evidence-based protocols in the context of support and expert consultation. Therapists appear to retain some interest in and commitment to maintaining these practices even after the external supports have been removed, but therapists appear to be selective in their maintenance of evidence-based practices. Future research and training curriculum development might benefit from identifying how best to support ongoing learning and maintenance of skills under typical practice conditions.

\section{References}

Aarons, G. A. (2004). Mental health provider attitudes toward adoption of evidence-based practice: The evidence-based practice attitude scale (EBPAS). Mental Health Services Research, 6, 61-74.

Beidas, R. S., Edmunds, J. M., Marcus, S. C., \& Kendall, P. C. (2012). Training and consultation to promote implementation of an empirically supported treatment: A randomized trial. Psychiatric Services, 63, 660665. doi:10.1176/appi.ps.201100401

Beidas, R. S., \& Kendall, P. C. (2010). Training therapists in evidencebased practice: A critical review of studies from a systems-contextual perspective. Clinical Psychology: Science and Practice, 17, 1-30. doi: 10.1111/j.1468-2850.2009.01187.x

Beidas, R. S., Koerner, K., Weingardt, K. R., \& Kendall, P. C. (2011). Training research: Practical recommendations for maximum impact. Administration and Policy in Mental Health and Mental Health Services Research, 38, 223-237. doi:10.1007/s10488-011-0338-z

Borntrager, C. F., Chorpita, B. F., Higa-McMillan, C., \& Weisz, J. R. (2009). Provider attitudes toward evidence-based practices: Are the concerns with the evidence or with the manuals? Psychiatric Services, 60, 677-681. doi:10.1176/appi.ps.60.5.677

Chu, B. C., Carpenter, A., Wyszynski, C., Conklin, P., \& Comer, J. (2014). Scalable options for extended skill building following didactic training in cognitive-behavioral therapy for anxious youth: A pilot randomized trial. Manuscript submitted for publication.

Cook, J. M., Schnurr, P., Biyanova, T., \& Coyne, J. (2009). Apples don't fall far from the tree: Influences on psychotherapists' adoption and sustained use of new therapies. Psychiatric Services, 60, 671-676. doi:10.1176/appi.ps.60.5.671

Craske, M. G., Farchione, T. J., Allen, L. B., Barrios, V., Stoyanova, M., \& Rose, R. (2007). Cognitive behavioral therapy for panic disorder and comorbidity: More of the same or less of more? Behaviour Research and Therapy, 45, 1095-1109. doi:10.1016/j.brat.2006.09.006

Darling-Hammond, L., \& Richardson, N. (2009). Teacher learning: What matters? How Teachers Learn, 66, 46-53.

David-Ferdon, C., \& Kaslow, N. J. (2008). Evidence-based psychosocial treatments for child and adolescent depression. Journal of Clinical Child and Adolescent Psychology, 37, 62-104. doi:10.1080/ 15374410701817865

Henggeler, S. W., Chapman, J. E., Rowland, M. D., Halliday-Boykins, C. A., Randall, J., Shackelford, J., \& Schoenwald, S. K. (2008). State- 
wide adoption and initial implementation of contingency management for substance-abusing adolescents. Journal of Consulting and Clinical Psychology, 76, 556-567. doi:10.1037/0022-006X.76.4.556

Herschell, A. D., Kolko, D. J., Baumann, B. L., \& Davis, A. C. (2010). The role of therapist training in the implementation of psychosocial treatments: A review and critique with recommendations. Clinical Psychology Review, 30, 448 -466. doi:10.1016/j.cpr.2010.02.005

Jensen-Doss, A., Hawley, K. M., Lopez, M., \& Osterberg, L. D. (2009). Using evidence-based treatments: The experiences of youth providers working under a mandate. Professional Psychology: Research and Practice, 40, 417-424. doi:10.1037/a0014690

Kazantzis, N., Whittington, C., \& Dattilio, F. (2010). Meta-analysis of homework effects in cognitive and behavioral therapy: A replication and extension. Clinical Psychology: Science and Practice, 17, 144-156. doi:10.1111/j.1468-2850.2010.01204.x

Kendall, P. C., \& Hedtke, K. A. (2006). Coping cat workbook (2nd ed.). Ardmore, PA: Workbook Publishing.

Miller, W. R., Yahne, C. E., Moyers, T. B., Martinez, J., \& Pirritano, M. (2004). A randomized trial of methods to help clinicians learn motivational interviewing. Journal of Consulting and Clinical Psychology, 72, 1050-1062. doi:10.1037/0022-006X.72.6.1050

Nakamura, B. J., Higa-McMillan, C. K., Okamura, K. H., \& Shimabukuro, S. (2011). Knowledge of and attitudes towards evidence-based practices in community child mental health practitioners. Administration and Policy in Mental Health and Mental Health Services Research, 38, 287-300. doi:10.1007/s10488-011-0351-2

Palinkas, L. A., Schoenwald, S., Hoagwood, K., Landsverk, J., Chorpita, B., \& Weisz, J. (2008). An ethnographic study of implementation of evidence-based treatments in child mental health: First steps. Psychiatric Services, 59, 738-746. doi:10.1176/appi.ps.59.7.738

Saitz, R., Sullivan, L. M., \& Samet, J. H. (2000). Training communitybased clinicians in screening and brief intervention for substance abuse problems: Translating evidence into practice. Substance Abuse, 21, 21-31. doi:10.1080/08897070009511415

Sholomskas, D. E., Syracuse-Siewert, G., Rounsaville, B. J., Ball, S. A., Nuro, K. F., \& Carroll, K. M. (2005). We don't train in vain: A dissemination trial of three strategies of training clinicians in cognitivebehavioral therapy. Journal of Consulting and Clinical Psychology, 73, 106-115. doi:10.1037/0022-006X.73.1.106

Silverman, W. K., Pina, A., \& Viswesvaran, C. (2008). Evidence-based psychosocial treatments for phobic and anxiety disorders in children and adolescents. Journal of Clinical Child and Adolescent Psychology, 37, 105-130. doi:10.1080/15374410701817907
Southam-Gerow, M. A., Rodriguez, A., Chorpita, B. F., \& Daleiden, E. L. (2012). Dissemination and implementation of evidence based treatments for youth: Challenges and recommendations. Professional Psychology: Research and Practice, 43, 527-534. doi:10.1037/a0029101

Southam-Gerow, M. A., Weisz, J. R., Chu, B. C., McLeod, B. D., Gordis, E. B., \& Connor-Smith, J. K. (2010). Does cognitive behavioral therapy for youth anxiety outperform usual care in community clinics? An initial effectiveness test. Journal of the American Academy of Child \& Adolescent Psychiatry, 49, 1043-1052. doi:10.1016/j.jaac.2010.06.009

Tosey, P. (1999). The peer learning community: A contextual design for learning? Management Decision, 37, 403-410. doi:10.1108/ 00251749910274171

Weisz, J. R., Chorpita, B. F., Palinkas, L. A., Schoenwald, S. K., Miranda, J., Bearman, S. K., . . Research Network on Youth Mental Health. (2012). Testing standard and modular designs for psychotherapy treatment depression, anxiety, and conduct problems in youth. Archives of General Psychiatry, 69, 274-282. doi:10.1001/archgenpsychiatry.2011 .147

Weisz, J. R., Southam-Gerow, M. A., Gordis, E. B., Connor-Smith, J. K., Chu, B. C., Langer, D. A., . . . Weiss, B. (2009). Cognitive-behavioral therapy versus usual clinical care for youth depression: An initial test of transportability to community clinics and clinicians. Journal of Consulting and Clinical Psychology, 77, 383-396. doi:10.1037/a0013877

Weisz, J. R., Thurber, C. A., Sweeney, L., Proffitt, V. D., \& LeGagnoux, G. L. (1997). Brief treatment of mild-to-moderate child depression using primary and secondary control enhancement training. Journal of Consulting and Clinical Psychology, 65, 703-707. doi:10.1037/0022-006X 65.4 .703

Weisz, J. R., Ugueto, A. M., Cheron, D. M., \& Herren, J. (2013). Evidencebased youth psychotherapy in the mental health ecosystem. Journal of Clinical Child and Adolescent Psychology, 42, 274-286. doi:10.1080/ 15374416.2013.764824

Wyman, P. A., Brown, H. C., Inman, J., Cross, W., Schmeelk-Cone, K., Guo, J., \& Pena, J. B. (2008). Randomized trial of a gatekeeper program for suicide prevention: 1-year impact on secondary school staff. Journal of Consulting and Clinical Psychology, 76, 104-115. doi:10.1037/0022006X.76.1.104

Received November 14, 2013 Revision received May 19, 2014 Accepted August 4, 2014 